\title{
Un viaje a las entrañas de la democracia
}

\author{
Rodolfo Humberto Aceves Arce
}

JoRge Alonso, 2012

La democracia de los de arriba en crisis

Universidad de Guadalajara, Guadalajara, 106 pp.

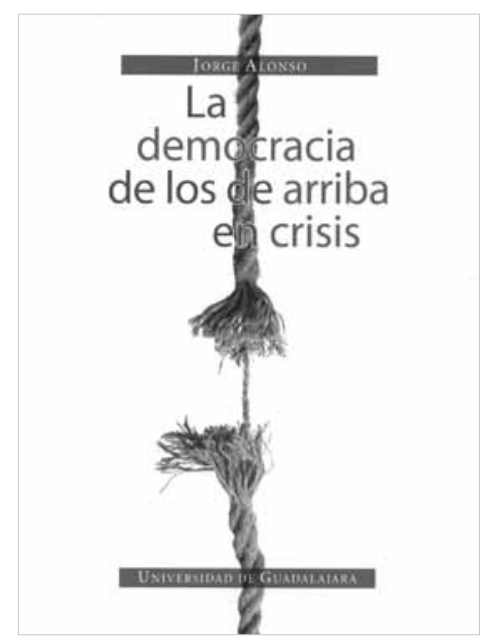

$\mathrm{E}$ ste libro es producto de la mirada antropológica de Jorge Alonso quien, en calidad de consejero ciudadano, vivió el proceso de reforma electoral en el estado de Jalisco en 1997. A pesar de situarse en un ámbito estatal que data de 15 años atrás, el tema cobra actualidad para el contexto nacional, en especial por el proceso electoral de 2012 y la alternancia de partido en la presidencia de la república.

\section{A Journey into the Bowels of Democracy}

Rodolfo Humberto Aceves Arce: Centro de Investigaciones y Estudios Superiores en Antropología Social-Occidente, Guadalajara, Jalisco, México rodo70@yahoo.com
Algunas de las experiencias y reflexiones vertidas en este texto proporcionan elementos para analizar la compleja realidad democrática mexicana actual. Alonso parte de la idea de que la democracia, en sus dimensiones intelectiva y práctica, es polisémica y multívoca. En tal sentido, Alonso recurre a dos concepciones de la democracia: la democracia de los de arriba, convertida en algo meramente formal por instituciones y sujetos, y la que es producto de sujetos que la hacen emanar desde abajo. El carácter antropológico de esta investigación se basa en la observación participante del autor en las consultas regionales y metropolitanas sobre la reforma electoral y en los debates en la Subcomisión de la Reforma 
Electoral. El sujeto de estudio fue el Consejo Electoral del Estado de Jalisco, formado por la cúpula del organismo electoral y por los representantes de los partidos políticos.

El libro está integrado por una introducción y cuatro capítulos en los que Alonso recorre la trayectoria de las reformas estatales en materia electoral y analiza el proceso de la reforma electoral de 1997. En el primer capítulo, "Prolegómenos de una reforma electoral", el autor revisa los principales procesos históricos de la legislación electoral del estado de Jalisco desde la década de 1910 hasta la de 1990 y vincula el contexto local con el nacional. En este espacio sobresalen la reforma política de 1977, las tendencias de la izquierda mexicana durante la década de 1970, el proceso electoral de 1988, la adopción del Código Federal de Instituciones y Procedimientos Electorales de 1990, el surgimiento del movimiento cívico en defensa del voto y en pro de la democratización, la ciudadanización de los órganos electorales y la alternancia en el gobierno estatal de Jalisco en 1994, cuando el Partido Acción Nacional (PAN) derrotó al Partido Revolucionario Institucional (PRI). De acuerdo con Jorge Alonso, para los organismos cívicos jaliscienses que pugnaban por elecciones libres y transparentes, este hecho representó una oportunidad para buscar cambios legislativos que fortalecieran una transición democrática a través de una nueva ley electoral y un organismo electoral confiable. Predominaba, pues, un ambiente político caracterizado por la esperanza de cambios en la forma de gobernar, debido a que los panistas habían sido críticos de los usos y costumbres de los priistas, quienes se encontraban en una situación difícil ante la pérdida de muchos puestos de elección popular.

En el capítulo "Discusiones para conseguir cambios en la legislación electoral", Jorge Alonso narra el inicio del proceso de generación de la reforma. En un primer momento, el gobernador Alberto Cárdenas impulsó la iniciativa de discusión organizada por los tres poderes estatales a finales de 1995, sin tomar en cuenta al organismo electoral ni a los presidentes de los partidos políticos. Esta situación provocó la protesta de partidos y del Consejo Electoral y que se pugnara por el lanzamiento de una nueva convocatoria para que fueran incluidos. De esta forma se originó una instancia temporal, la Subcomisión de la Reforma Electoral, la cual se encargaría de realizar foros de consulta sobre partidos y elecciones. En un principio quedó conformada por los consejeros ciudadanos y los representantes de los partidos en el Congreso de la entidad: PAN,
PRI y Partido de la Revolución

Democrática (PRD), y

posteriormente se incorporaron

los representantes de partidos que mantenían su registro: Partido del Trabajo (РT), Partido Verde Ecologista de México (PVEM) y Partido del Frente Cardenista de Reconstrucción Nacional (PFCRN). La Subcomisión convocó a partidos, instituciones educativas, colegios de profesionistas, investigadores y estudiosos del ámbito electoral, organizaciones no

gubernamentales, asociaciones, medios de comunicación y ciudadanos en general a participar en seis foros regionales entre abril y junio de 1996 en Lagos de Moreno, Ciudad Guzmán, Puerto Vallarta, Colotlán, Autlán y la Zona (ZMG). En los foros la discusión se centró en siete temas principales: los principios rectores, los órganos y los procedimientos electorales; las condiciones de competencia electoral; la representación política; las asociaciones políticas y las coaliciones; la jornada electoral; el presupuesto y patrimonio de los organismos electorales; el servicio profesional electoral y la promoción y difusión de la cultura cívico-electoral.

El autor realiza un análisis detallado de las consultas y de los trabajos de la Subcomisión. Las labores de esta instancia se llevaron a cabo entre junio de 
1996 y septiembre de 1997 e incluyeron las discusiones sobre el material de los foros y su organización en temas. Alonso muestra minuciosamente las proporciones de los temas de las ponencias en las regiones, de los tipos de participantes en las regiones y en la ZMG, de la participación por partidos, así como las propuestas nodales de éstos, sus intervenciones en las discusiones, las etapas del trabajo de la Subcomisión y los principales puntos debatidos en ella. Cabe resaltar que este trabajo analítico se basa en los registros del diario de campo del autor. Asimismo, Alonso vincula las discusiones de los foros en materia electoral con los de otros reforma del Poder Ejecutivo y la política social - mayo a junio de 1996-, a la reforma del Poder Legislativo — agosto y septiembre de 1997- y a la reforma municipal. Al final de este capítulo, el autor contrasta el entusiasmo del gobierno de Jalisco por la participación ciudadana sin precedentes en las discusiones de la reforma integral del Estado con su percepción sobre este proceso. Señala que la participación cívica fue escasa y que la mayoría de las ponencias en los foros provenía de la clase política y de sus allegados, por lo cual los intereses ciudadanos de abajo no eran tomados en cuenta.

El capítulo "La reforma electoral y de participación

ciudadana en Jalisco a finales del siglo $\mathrm{xx}$ " inicia con la narración de los últimos trabajos de la Subcomisión, en específico los debates finales y la redacción de su propuesta antes de ser enviada a una comisión política integrada por representantes de los tres poderes a finales de enero de 1997. En la propuesta en material electoral destacaban avances como los relacionados con las figuras del referéndum, el plebiscito y la iniciativa popular, así como puntos problemáticos, como las proporciones para lograr acuerdos en el Congreso, el voto de los representantes de los partidos en el organismo electoral, la reconformación del Poder Judicial, la integración del Consejo Electoral y el número de diputados de mayoría relativa y de representación proporcional. En este apartado Alonso también se refiere a las dificultades que ponían en peligro la reforma: sucesos locales, como la represión a tianguistas priistas que tomaron el palacio municipal de Guadalajara en el marco de la campaña electoral federal y suscitaron diferencias entre los partidos políticos estatales. Enseguida, el autor compara los resultados de la Subcomisión de la Reforma Electoral con la propuesta que envió el Ejecutivo estatal al Congreso los últimos días de febrero de 1997, en forma de iniciativa de reformas y adiciones a la Constitución Política de Jalisco. A su vez, detalla el complejo proceso de negociaciones que culminó con la aprobación en el Congreso de la nueva legislación electoral a finales de abril de 1997 y las relaciones entre la reforma electoral jalisciense y la legislación electoral federal de 1996.

Otro punto destacado es la evaluación del autor entre lo legislado y las propuestas de la Subcomisión, de las organizaciones ciudadanas y de las demandas de los foros. Su apreciación es que los partidos lograron que las condiciones de competencia se ajustaran a su interés en detrimento del de los ciudadanos como sujetos políticos plenos. Una muestra de este fenómeno son los ejercicios de las figuras de participación ciudadana: el referéndum, el plebiscito y la iniciativa popular. En el contexto nacional, Jalisco fue el tercer estado en admitir estas formas de participación ciudadana en 1997. El autor ofrece ejemplos que exponen los fracasos al intentar aplicarlas, en especial el efecto de candados impuestos a estos instrumentos por la clase política hacia los ciudadanos jaliscienses. Como lo expresa Alonso: "La clase política ha demostrado que no le gusta la participación ciudadana, y que cuando ésta aparece, recibe trabas de todo tipo para que no consiga sus fines".

El último capítulo, "Balance y una reflexión final”, es un esfuerzo de síntesis del proceso 
descrito en los apartados anteriores que resulta fundamental por la experiencia de Alonso como consejero ciudadano de la Subcomisión. El autor retoma sus observaciones para reflexionar sobre las formas en que la participación ciudadana es manipulada por la clase política. Para el caso de Jalisco, los de arriba impusieron los temas de las consultas y las dinámicas bloquearon los debates entre los participantes, además de que una gran proporción de éstos estaban relacionados con partidos políticos y burocracia. En este sentido, los partidos lograron el control de la Subcomisión y de los instrumentos de participación ciudadana. También destaca el carácter asimétrico de las relaciones entre partidos y ciudadanos $\mathrm{y}$ entre partidos grandes $\mathrm{y}$ partidos pequeños. La traducción de estas dinámicas y relaciones desiguales es una democracia impuesta desde arriba para conservar la dominación y la opresión y para hacer creer que los intereses de unos pocos son los intereses de todos.

Alonso introduce el término crisis para comprender la realidad política mexicana. Para él, esta crisis ha transparentado una vinculación entre una partidocracia y los poderes fácticos del gran dinero, del narcotráfico, de la alta jerarquía eclesiástica católica y, en especial, de los grandes medios de comunicación electrónicos. Ha sido una relación en la que los partidos se han supeditado a los poderes fácticos y que ha favorecido el surgimiento y fortalecimiento de una dictadura camuflada con el consecuente fracaso de la democracia de los de arriba. Por ello, para Alonso los partidos se han alejado de los intereses ciudadanos y se han ocupado de sus intereses corporativos al servicio de los poderes fácticos. Así los ciudadanos, más que participantes, son espectadores-consumidores de la política.

Otra idea sugerente es la de considerar la crisis política mexicana como una combinación de totalitarismo invertido con fascismo societal. Alonso se auxilia de las propuestas de Sheldon Wolin y Boaventura de Sousa Santos. El totalitarismo invertido, de acuerdo con Sheldon Wolin, es un sistema de poder opuesto a los principios fundamentales de la democracia que dispone de tecnologías de control, intimidación y manipulación masiva más sofisticadas que las del totalitarismo clásico. Se enfoca en el poder corporativo y en la desmovilización política ciudadana. Además, explota recursos estatales y genera dinámicas con el poder de corporaciones económicas. Se caracteriza asimismo por no expresarse como ideología, pero sí como productor de una mercantilización de la política y de la religión. Por otro lado, el rasgo distintivo del fascismo societal, según Boaventura de Sousa Santos, son las relaciones sociales y experiencias de vida bajo relaciones de poder e intercambios desiguales que no implican un regreso del fascismo clásico, pero que sí se dirigen a formas de exclusión severas y potencialmente irreversibles. Sin sacrificar los aspectos formales de la democracia, el fascismo societal produce una sociedad civil profundamente estratificada. Con base en estas dos propuestas, el autor advierte cómo en el contexto mexicano una parte de la población se supedita a los intereses de los de arriba por medio de manipulación mediática, política y electoral.

Alonso echa mano de diversos estudios de mediciones sobre democracia para demostrar que la gran mayoría de mexicanos no tiene confianza en la democracia de los de arriba. Aunados a estos indicadores de la crisis de esta democracia particular, el autor señala algunos signos del deterioro político mexicano: los recortes legislativos de recursos a sectores clave como la educación, la salud, el combate a la pobreza y la infraestructura, sin mencionar la enorme cantidad destinada a los partidos políticos — una clase política parasitaria puesta al servicio de los poderes fácticosy la colocación de los poderes fácticos, principalmente de los 
grandes medios de comunicación electrónicos, por encima de los poderes constitucionales. A decir de Alonso, la crisis de la democracia de los de arriba también puede constatarse en el grave descrédito en que han caído, ya que grandes sectores sociales no confían en la relación de la partidocracia con los poderes fácticos. En este punto, el autor detecta dos perspectivas complementarias de esta crisis: una que es producto de las formas de utilización cínica de la política para los intereses de los partidos y de los poderes fácticos, y otra que es producto de los de abajo, quienes se han desvinculado y actúan por su propia cuenta. De esta forma, la democracia de los de arriba ha perdido paulatinamente sustento entre los ciudadanos.

En este libro Jorge Alonso nos ofrece elementos para analizar y caracterizar el sistema político mexicano a partir de una experiencia particular y claramente localizada. El desmenuzamiento del proceso de la reforma electoral jalisciense de 1997 confiere solidez y profundidad a esta obra. Hay que tomar en cuenta que el autor participó activamente en este proceso como consejero ciudadano, pero también como antropólogo social. Por tal motivo, este documento representa una fuente valiosa para adentrarnos en contextos en los que se diseña la política y para comprender las formas de apropiación de la democracia por parte de la clase política, así como de los mecanismos de exclusión de la participación ciudadana. Por último, este libro también puede proporcionar puntos de partida para buscar alternativas de hacer política desde los de abajo. Por esta razón, espero que su estudio sobre la democracia de los de abajo, anunciado en esta obra, salga pronto de los talleres de impresión. Nuestra sociedad requiere de otras alternativas de hacer política que nos acerquen al ejercicio de una ciudadanía plena. 